\title{
Segmentation of Curvilinear Objects using a Watershed-Based Curve Adjacency Graph
}

\author{
Thierry Géraud \\ EPITA Research and Development Laboratory, \\ 14-16 rue Voltaire, F-94276 Le Kremlin-Bicêtre cedex, France, \\ thierry.geraud@lrde.epita.fr, \\ WWW home page: http://www.Irde.epita.fr/
}

\begin{abstract}
This paper presents a general framework to segment curvilinear objects in 2D images. A pre-processing step relies on mathematical morphology to obtain a connected line which encloses curvilinear objects. Then, a graph is constructed from this line and a Markovian Random Field is defined to perform objects segmentation. Applications of our framework are numerous: they go from simple surve segmentation to complex road network extraction in satellite images.
\end{abstract}

\section{Introduction}

Many different methods have been proposed to segment curvilinear structures in 2D images. Let us just recall some of them which are, to our humble opinion, the most promising ones:

- tracking by active testing by Geman and Jedynak (1996);

- unifying snakes, region growing and energy/Bayes/MDL, so-called region competition, by Zhu and Yuille (1996);

- defining Markovian field on a set of segments by Tupin et al. (1998);

- dynamic programming for saliency optimization by Lindenbaum and Berengolts $(2000)$;

- using a Markov point process by Stoica et al. (2000).

These methods suffer from drawbacks. The saliency approach does not rely on a global optimization process. Tracking-like approaches cannot plainly take into account features extracted from image regions and require a starting point; these approaches are thus limited to rather easy segmentation problems. Markovian approaches are often computationally expensive due to the high number of primitives - small segments - they have to handle. Last, region competition is also an expensive approach where both the region and variational flavors are not often pertinent when objects are not regions.

In this paper we propose a general framework for curvilinear object segmentation that overcomes these drawbacks.

This paper is organized as follows. The first section is a preliminary section that introduces the basic ideas and tools on which the proposed framework relies. Section 3 then describes the framework itself and illustrates its capabilities on road extraction in satellite images; afterwards, we conclude in section 4 . 


\section{Preliminaries}

\subsection{Watershed Transform}

The watershed transform (WT), as explained by Vincent and Soille (1991), is a morphological algorithm usually used for the purpose of segmentation. Considering a gray level image as a topographic map, let us denote by catchment basin associated with a regional minimum of this map, all points whose steepest slope paths reach this minimum. The watershed line is a closed one-pixel thick crest line which separates every adjacent catchment basins, i.e., regions.

\subsection{Region Adjacency Graph and Markov Random Field}

A now common framework to segment an image $I$ or to extract objects from $I$ is based on the watershed transform; it can be summarized as follows.

1. An image $G$ of the gradient norm of $I$ is computed. Contours in the gradient norm image (GNI) $G$ have high intensity values whereas regions have low intensity values.

2. The watershed transform (WT) is applied to $G$ which results in getting a partition of $I$ into regions. The watershed line passes through crest lines of $G$, that is, objects contours. This partition, $P$, is an over-segmentation since $G$ contains a number of minima greater than the effective number of objects/regions to segment.

3. The region adjacency graph (RAG) is extracted from $P$. A node corresponds to a region (more precisely, a catchment basin) and an edge between two nodes indicates that these regions are adjacent. Extra information are put into the graph; for instance they can be statistical estimations concerning regions of $I$ which are then enclosed in graph nodes, or saliency values of contours estimated from $I$ and added to graph nodes.

4. The last step aims at grouping regions according to given criterions in order to get a final segmentation. To that aim, a Markov Random Field (MRF) is defined onto the RAG and the segmentation process is handled by a Markovian relaxation.

This framework is powerful since it remains general - it can be applied to various imagery segmentation problems - and since the final segmentation results from a global process on high-level image primitives (regions in that case). Moreover, it enables operational segmentations even when images are over-sized and when objects are difficult to segment; for instance, Géraud et al. (1995) succeed in segmenting internal brain structures from magnetic resonance images. Let us mention that this framework has been discussed by many authors such as Kim and Yang (1994); Haris et al. (1998); Bleau and Leon (2000); Sarkar et al. (2000), and a multi-scale version of this framework has been proposed by Gauch (1999). 


\subsection{Minima Suppression and Area Closing}

A classic algorithm to suppress minima in images is the morphological closing operator. When these is no prior information about the shape of image objects, closing is usually performed with a structural element being a disk in order to preserve isotropy. However, artifacts appear in resulting images: in particular, crest lines can strongly move when one wants to remove many minima, that is, when filtering strength (i.e., the disk radius) increases.

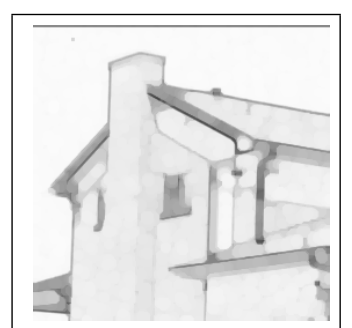

(a) Closing of GNI with a Disk $(r=4,3)$

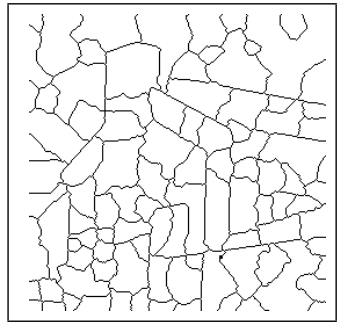

(c) $W$ obtained from image (a)

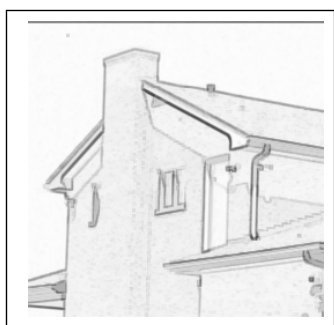

(b) Area Closing of GNI $(a=50)$

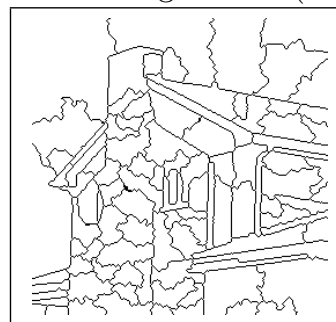

(d) $W$ obtained from image (b)

Fig. 1. Watershed Transform Results with the Same Final Number of Regions.

Conversely, an area closing operator does not present this drawback. This operator is a "connected filter", as described by Salembier and Serra (1995), which removes minima whose area (influence zone) is less than a given threshold. A fast implementation of this operator is provided by Meijster and Wilkinson (2002).

Figure 1 illustrates the contour shifting / un-shifting properties of both "classical" and area closing operators. Starting from the classical HOUSE image, we apply closing operators to its gradient norm image (GNI); the negatives of the results are depicted by images (a) and (b). We then apply the watershed transform algorithm, which respectively leads to images (c) and (d). Please note that these segmentation results contain the same number of regions. However, contours are shifted when the classical closing is involved which is not the case with the area closing. Moreover, in the former case regions have more uniform sizes and are spread more uniformly over the image space than in the latter case. This is another drawback since we prefer segmentations that are more adapted to original image data. 


\section{Proposed Framework}

Although region-based methods are not well suited to segment curvilinear objects, we now propose a framework which relies on a region segmentation algorithm to address this issue.

\subsection{Framework Description}

Our framework is very similar to the one described in section 2.2 .

Pre-Processing. From an original image containing curvilinear objects we compute a gray level image where pixel values denote their potential of belonging to these objects. Curvilinear objects are thus located on some parts of the crest lines of this "potential" image.

Morphological Filtering. The filtering step consists in computing an area closing of the potential image and then running the watershed transform. The "closed" potential image has much less minima than the "original" potential image while properly retaining crest lines location (Cf. discussions of sections 2.1 and 2.3). Therefore, the resulting watershed line includes the curvilinear objects.

Curve Adjacency Graph. From the watershed line, we build a curve adjacency graph (CAG). A node of this graph (red bullets in the picture below) represents a shed, that is, a connected part of the watershed line separating two adjacent basins. An edge (green lines in the picture below) is drawn between two nodes/sheds if one end of the first shed is connected with a end of the second one through the watershed line.

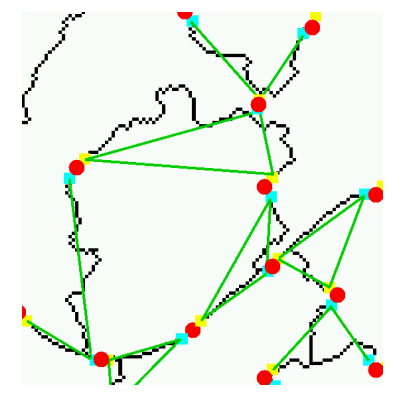

For every node we make the distinction between edges coming from connections to one shed end (yellow anchors) and those coming from connections to the other shed end. This distinction is symbolized by yellow and blue anchors in the picture above.

Markovian Relaxation Segmenting curvilinear objects now turns out to be a graph labeling problem. Upon the graph structure, we define a Markov random field. Let us denote by $X$ the observation field, by $Y$ the result field, by $x_{s}$ and $y_{s}$ their respective restriction to a given node $s$, by $Y_{V_{s}}$ the restriction of $Y$ to 
the neighborhood of $s$. The variable $y_{s}$ has a Boolean realization where 1 means object and 0 means not object. Under the Markovian assumption we have:

$$
p\left(y_{s} \mid X, Y-y_{s}\right)=\frac{1}{Z} \exp \left(-\left(U\left(x_{s}, y_{s}\right)+U\left(Y_{N_{s}}\right)\right)\right) .
$$

The first energy term, $U\left(x_{s}, y_{s}\right)$, models a priori knowledge about curvilinear objects, and the second energy term, $U\left(Y_{N_{s}}\right)$ deals with labeling contextual information. Since we have expressed the object segmentation problem as an energy minimization problem, a relaxation process is performed to finally get the segmentation result.

\subsection{Framework Adaptation}

In order to apply this framework to a given segmentation application, this framework should be adapted.

The first step depends on the particular application and on the original image data. For instance, when the original image contains a curve to be segmented and when this curve is dark pixels on white background, the potential image can be as simple as the original image once inverted. An other example is the case of road network extraction from a multi-channels satellite image; then the proper channels should be processed (fused) to build the potential image.

Setting the area parameter of the morphological filtering step also depends on both application and data. As explained in section 2.3, this parameter removes image local minima. Thus, considering the watershed transform result, this parameter has an effect of merging small catchment basins. When a curvilinear object contains a loop, this loop can disappear if its area is lower than the area parameter value.

Last, defining the energies for the Markov random field is also data dependent. Features associated with nodes - a priori knowledge about piece of curvilinear objects - are numerous; they can be the potential mean value along the piece of curve, a curvature measurement, its saliency as discussed by Najman and Schmitt (1996), and so on. Features related to contextual energy express knowledge about the global shape of the curvilinear objects and the connections between its different parts; for instance, a feature can be a continuity measure when the object is a smooth curve or, in the contrary, a measure that ensures that the object is only composed of straight lines and $\pi / 2$ breaks.

\subsection{Illustration}

We have applied our framework to different image segmentation issues. In this section, we present a result in the field of road extraction network. It is illustrated with a small part $(700 \times 380$ pixels $)$ of a Landsat image from St-Johns city, Canada, having a $25 \mathrm{~m}$ resolution and 7 spectral channels; see figure 2 . This original image is under "Copyright (C) 2000. Government of Canada with permission from Natural Resources Canada" (http://geogratis.cgdi.gc.ca/). Applying the whole road extraction process to an image having $2.10^{6}$ pixels takes less than 
20s on a 1,7 GHz personal computer running GNU/Linux and using our image processing library Olena (Cf. section "notes and Comments" after section 4) which provides fast implementation of algorithms.

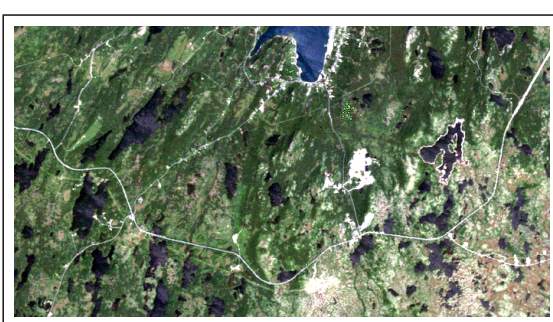

(a) Original Image in Natural Colors

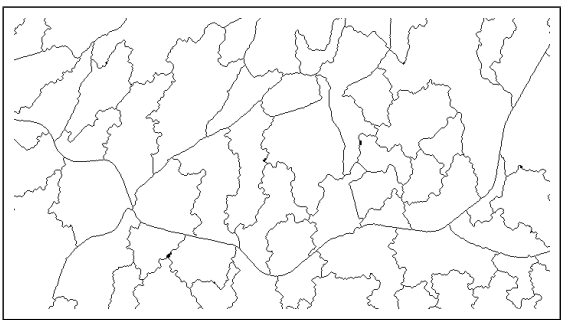

(c) Watershed Line when $a=500$

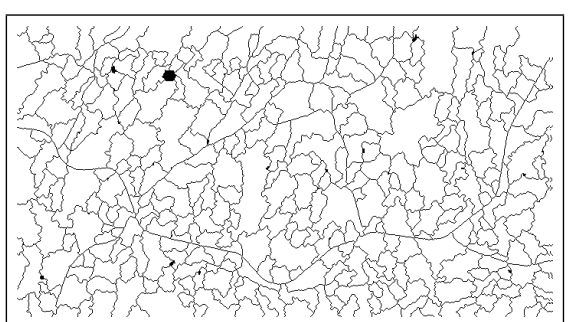

(b) Watershed Line when $a=50$

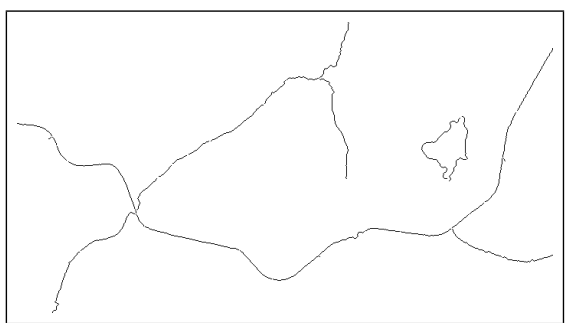

(d) Final MRF Labeling

Fig. 2. Application to Road Network Extraction.

As one can see on figures 2 (b) and 2 (c), with different values of the area parameter the resulting watershed line is more or less simplified but data of interest are not affected. Extra information about applying the proposed method to road network extraction are given in Géraud (2003).

\section{Conclusion}

We have presented a method to extract road network from satellite images. We have transposed the recognition scheme "WT + RAG + MRF", described in section 2.2 and which is dedicated to image segmentation, to the problematic of road network recognition. To that aim, we propose a recognition scheme that is, as far as we know, original: "area opening $+\mathrm{WT}+\mathrm{CAG}+\mathrm{MRF}$ ".

This recognition scheme is a global optimization process so it provides robust and reproducible results. Moreover, it is general and can easily be adapted to various image processing fields where the recognition of curvilinear structures is involved.

Notes and Comments. Source code of our method is available on the Internet from the location http://www.lrde.epita.fr. It has been developed using 
OLENA, our generic image processing library. OLENA is free software under the GNU Public Licence (GPL) and information about this library are presented by Darbon et al. (2002). 


\section{Bibliography}

A. Bleau and L. Leon. Watershed-based segmentation and region merging. Computer Vision and Image Understanding, 77:317-370, 2000.

J. Darbon, T. Géraud, and A. Duret-Lutz. Generic implementation of morphological image operators. In Mathematical Morphology, Proceedings of the 6th International Symposium VI (ISMM), pages 175-184, Sydney, Australia, April 2002. Sciro Publishing.

J. Gauch. Image segmentation and analysis via multiscale gradient watershed hierarchies. IEEE Trans. on Image Processing, 8(1):69-79, January 1999.

D. Geman and B. Jedynak. An active testing model for traking roads in satellite images. IEEE Trans. on Pattern Analysis and Machine Intelligence, 18(1): $1-14,1996$.

T. Géraud. Fast road network extraction in satellite images using mathematical morphology and Markov random fields. In Proceedings of the IEEE-EURASIP Workshop on Nonlinear Signal and Image Processing, Grado, Italy, June 2003. To appear.

T. Géraud, J.-F. Mangin, I. Bloch, and H. Maître. Segmenting internal structures in 3D MR images of the brain by Markovian relaxation on a watershed based adjacency graph. In Proc. of the IEEE Int. Conf. on Image Processing, volume 3, pages 548-551, 1995.

K. Haris, S. Efstratiadis, N. Maglaveras, and A. Katsaggelos. Hybrid image segmentation using watersheds and fast region merging. IEEE Trans. on Image Processing, 7(12):1684-1699, December 1998.

I. Kim and H. Yang. A systematic way for region-based image segmentation based on Markov random field model. In Pattern Recognition Letters, volume 15, pages 969-976, October 1994.

M. Lindenbaum and A. Berengolts. A probabilistic interpretation of the saliency network. In Proceedings of the European Conference on Computer Vision (2), volume 1843 of Lecture Notes in Computer Science, pages 257-272. Springer, 2000.

A. Meijster and M. Wilkinson. A comparison of algorithms for connected set openings and closings. IEEE Trans. on Pattern Analysis and Machine Intelligence, 24(4):484-494, 2002.

L. Najman and M. Schmitt. Geodesic saliency of watershed contours and hierarchical segmentation. IEEE Trans. on Pattern Analysis and Machine Intelligence, 18(12):1163-1173, December 1996.

P. Salembier and J. Serra. Flat zones filtering, connected operators, and filters by reconstruction. IEEE Trans. on Image Processing, 4(8):1153-1160, 1995.

A. Sarkar, M. Biswas, and K. Sharma. A simple unsurpervised MRF model based image segmentation approach. IEEE Trans. on Image Processing, 9(5): 801-812, May 2000.

R. Stoica, X. Descombes, and J. Zerubia. A Markov point process for road extraction in remote sensed images. Technical Report 3923, INRIA, 2000. 
F. Tupin, H. Maître, J.-F. Mangin, J. Nicolas, and E. Pechersky. Detection of linear features in SAR images: Application to road network extraction. IEEE Trans. on Geoscience and Remote Sensing, 36(2):434-453, 1998.

L. Vincent and P. Soille. Watersheds in digital spaces: an efficient algorithm based on immersion simulations. IEEE Trans. on Pattern Analysis and Machine Intelligence, 13(6):583-598, June 1991.

S. C. Zhu and A. Yuille. Region competition: Unifying snakes, region growing, energy/bayes/MDL for multi-band image segmentation. IEEE Trans. on Pattern Analysis and Machine Intelligence, 18(9):884-900, September 1996. 